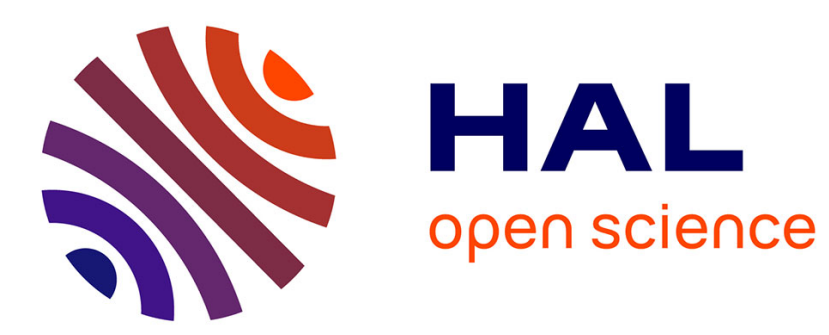

\title{
Territoires musicaux de la samba
}

Alessandro Dozena

\section{To cite this version:}

Alessandro Dozena. Territoires musicaux de la samba. L'Information géographique, 2018, 10.3917/lig.824.0084 . hal-02105294

\section{HAL Id: hal-02105294 \\ https://hal.science/hal-02105294}

Submitted on 19 May 2019

HAL is a multi-disciplinary open access archive for the deposit and dissemination of scientific research documents, whether they are published or not. The documents may come from teaching and research institutions in France or abroad, or from public or private research centers.
L'archive ouverte pluridisciplinaire HAL, est destinée au dépôt et à la diffusion de documents scientifiques de niveau recherche, publiés ou non, émanant des établissements d'enseignement et de recherche français ou étrangers, des laboratoires publics ou privés. 


\title{
TERRITOIRES MUSICAUX DE LA SAMBA
}

\section{MUSICAL TERRITORIES OF SAMBA}

\author{
Dozena, Alessandro \\ Universidade Federal do Rio Grande do Norte, Natal (Brésil) \\ Chercheur Invité, Université Paul Valéry, Montpellier (France) ${ }^{1}$ \\ sandozena@gmail.com \\ Contact Postal: Avenida das Américas 1342 casa 301 \\ Parque das Nações, Parnamirim - RN, Natal, Brésil
}

CEP 59158-150

\begin{abstract}
Résumé
Le but de cet article est d'analyser les territoires musicaux de la samba présents dans certaines villes brésiliennes et européennes. Celui-ci se manifeste par des tactiques quotidiennes qui révèlent la capacité utopique et inventive des sujets participants. Pour atteindre cet objectif, nous réfléchirons avec plus d'attention sur les pratiques culturelles suivantes : le carnaval de São Paulo, les territoires de la samba à São Paulo, les territoires de la samba à Europe: spécifiquement sur le Samba Festival (Allemagne-Coburg), le Lavage de la Madeleine (ParisFrance) et le carnaval de La Grande Motte (La Grande Motte-France). La mise en oeuvre de notre proposition est passée par la réalisation d'entrevues semi-structurées avec les sujets impliqués (producteurs culturels, directeurs d'écoles de samba, musiciens brésiliens et français appartenant à des Batucadas).
\end{abstract}

Mots-clés: Samba, territoires musicaux, Brésil, Europe.

Abstract

The purpose of this article is to analyze the musical territories of samba present in some brazilian and european cities. It manifests itself in daily tactics that reveal the utopian and inventive capacity of the participants. To achieve this goal, we will think more carefully about the following cultural practices: the São Paulo carnival, the samba territories in São Paulo, the samba territories in Europe: specifically on the Samba Festival (Germany-Coburg ), the Wash of the Madeleine (Paris-France) and the Carnival of La Grande Motte (La Grande MotteFrance). The implementation of our proposal was carried out through semi-structured interviews with the subjects involved (cultural producers, directors of samba schools, brazilian and french musicians belonging to Batucadas).

Key-words: Samba, musical territories, Brazil, Europe

\footnotetext{
${ }^{1}$ Bourse CAPES Processus BEX 0663/15-8. Les réflexions développées ici font partie d'une recherche menée lors d'un stage post-doctoral au Département de Géographie de l'Université Paul Valéry Montpellier / France, laboratoire ART-Dev UMR 5281. Merci beaucoup à Alix Macadré pour la relecture de la première version du texte et à Dominique Crozat pour la collaboration.
} 
Cet article entend proposer une réflexion sur le genre musical samba, « consommé » non seulement par les brésiliens, mais aussi par les étrangers. On sait que la samba, danse et genre musical, est vivante au Brésil et ailleurs, et qu'elle traverse différentes générations et frontières sociales, en s'adaptant aux changements et en s'affirmant comme un élément important de la culture brésilienne, notamment après son inscription, en 2004, en tant que patrimoine immatériel par l'Institut du Patrimoine Historique et Artistique National (IPHAN), organisme fédéral chargé de la protection du patrimoine brésilien. De plus, en 2005, l'United Nations Organization for Educational, Science and Culture (UNESCO) a reconnu la Samba de Roda dans le Recôncavo Bahianais comme patrimoine immatériel de l'humanité.

La samba est plus qu'un genre de musique et de danse, car elle exprime des façons de penser, de sentir et de construire des formes privées de territoires musicaux. Les pratiques, les discours et les représentations des sambistas font passer un message anti-utilitariste, contraire à la logique productiviste, prônant l'imagination, la sensibilité, la ludicité et la transgression.

Dans cet article, il s'agira de proposer une réflexion sur les territoires musicaux de la samba présents au Brésil et en Europe, à partir de la cartographie conçue et de la réalisation d'entretiens semi-directifs auprès des individus impliqués: musiciens brésiliens et européens appartenant à des batucadas, producteurs culturels et directeurs d'écoles de samba. Il est à noter que l'école de samba est une association culturelle et sociale dont les membres se rassemblent dans le but d'organiser des activités liées à la samba.

Ces territoires musicaux se manifestent par des tactiques quotidiennes qui révèlent la capacité utopique et inventive des participants. Il s'agira donc ici de réfléchir sur les territoires musicaux de la samba à São Paulo et du Samba Festival (AllemagneCoburg). 


\section{Les Territoires de la Samba dans la Métropole Mondiale Brésilienne}

L'exemple de la ville de São Paulo montre que la samba agit comme un élément perturbateur de l'ordre établi, s'érigeant contre un esprit productiviste ambiant; elle redonne une certaine magie à la vie, qu'elle rend moins routinière, moins mécanisée et moins administrée par les pouvoirs publics. On verra que cette idée dialogue avec ce qui se trouve sur les territoires musicaux de la samba en Europe, et d'une certaine façon, avec la théorie des jeux et ambigüités de la construction musicale des identités spatiales (Crozat, 2016), avec les concepts de déviation (Certeau, 1997) et de rationalités autres (Santos, 2002).

Dans Dozena (2012) nous avions cherché à mettre en lumière les aspects distinctifs de la territorialisation de la samba à São Paulo et comprendre certains processus par lesquels la samba établit des territorialités par le biais de pratiques sociales et de représentations subjectives qui font de São Paulo une ville capable d' «inviter» les individus à la réalisation humaine grâce à la force intégratrice de la samba, qui imprègne tous les quartiers. Pour cela, il était nécessaire de comprendre la signification des territoires délimités par les sambistas et d'analyser l'interaction des sambistas avec ces territoires.

La notion de sambista qui est considérée ici, englobe le sujet qui conduit l'«évolution» de la samba avec ses propres actions et motivations, guidées par les choix et conceptions propres au sambista et qui impliquent des discours, des représentations et des formes de consciences particuliers. Les sambistas sont des personnes qui réalisent la samba (danseurs de samba, musiciens de samba ou amateurs de samba) et qui participent aux activités liées à la samba (carnaval, fêtes des écoles de samba et cercles de samba). Les cercles de samba agissent comme des territoires musicaux qui génèrent des interactions sociales et un certain sens d'appartenance à une communauté; et s'expriment littéralement par la samba pratiquée dans un cercle.

L'une des réflexions importante concerne le fait que la samba n'est pas juste un style musical et qu'elle revêt encore dans l'imaginaire social du brésilien et de l'étranger une des plus importantes formes de représentation de brazilitude. Il faut reconnaître que la samba dépasse le simple style musical et que certaines relations 
établies par les classes sociales populaires dans leurs quartiers ne sont généralement pas révélées à toute la société.

Au Brésil, la samba revêt une plus grande dimension que le carnaval. Dans le cas spécifique de São Paulo, bien que la fête du carnaval ait été relayée par des émissions de télévision (notamment les évènements se déroulant au Sambodrome ${ }^{2}$ ), elle n'a jamais quitté les quartiers. Au sein de ces derniers, nous trouvons les cercles de samba, les blocs de carnaval et les mouvements de samba; en plus des événements qui ont lieu toute l'année dans les écoles de samba.

Cela dit, il est bon de différencier le carnaval de la samba au Brésil, carnaval qui trouve son apogée au cours des fêtes qui ont lieu en février ou mars et qui est marqué par l'appropriation de certains éléments présents dans la samba. Il est également important de réaffirmer que l'univers de la samba englobe non seulement un style musical, mais aussi des pratiques socio-spatiales et des modes de vie qui coexistent avec les événements carnavalesques; ce qui a influencé historiquement sa configuration et son association directe avec le carnaval.

Une autre constatation est que, de plus en plus, les mouvements et les cercles de samba dialoguent avec la communauté en concevant la sociabilité comme une prémisse fondamentale. En ce sens, ils s'opposent, dans la plupart des cas, aux valeurs culturelles de la consommation immédiate, entrevoyant de nouvelles possibilités notables de construction d'autres réalités.

Surtout après la décennie de 1990, la ville de São Paulo a connu une «vague revitalisante » impulsée par les mouvements de samba qui cherchaient à sauver les « racines » de la samba et à s'unir avec les sambistas du passé.

Dans leur engagement direct avec la samba, communautairement, les sambistas s'approprient des territoires à la fois pour la production et la reproduction de leur vie matérielle et pour l'expérience territoriale. Ce sont ces appropriations de l'espace géographique, qui, à partir des relations sociales, produisent et peuvent renforcer une identification qui utilise le territoire comme une référence. Ainsi, les sambistas développent des échanges symboliques avec les territoires dans lesquels ils vivent,

\footnotetext{
${ }^{2}$ Le Sambadrome de Anhembi est un espace géré par la Mairie de São Paulo, où la fête carnavalesque acquiert une dimension spectacularisée, étant diffusée par la principale chaine de télévision au Brésil, le réseau Globo de télévision.
} 
"faisant partie de leur équipement psychique, acquis dans leur processus de socialisation et de vie » notre traduction ${ }^{3}$ (Moraes, 2001, p. 44).

Cherchant à interpréter les représentations et identifications présentes sur le territoire et en concevant celui-ci comme une dimension de l'expérience humaine des lieux, nous avons considéré l'appropriation effectuée quotidiennement par les groupes qui l'habitent et lui donnent une dimension non seulement symbolique, mais aussi économique et politique. En abordant les territorialités dans «le monde de la samba » à São Paulo, nous considérons que l'appropriation «peut être construite à partir de multiples véhicules, imaginaires, sentiments, biens, propriétés, utilisations, et sans laquelle aucun d'entre eux ne signifie toujours l'exercice effectif du contrôle sur les objets et les pratiques sociales qui se produisent» notre traduction (Gomes, 2002, p.13) ${ }^{4}$.

La désignation «monde de la samba » est destinée à englober les activités qui ont pour élément central la samba, notamment celles qui se déroulent dans les écoles de samba, dans les cercles de samba, dans les bars, les discothèques ou au travers de tout projet de samba. En raison de l'amplitude des possibilités de recherche, nous avons choisi de nous concentrer sur une analyse au sein des écoles de samba, cercles et mouvements de samba. Nous ne nous sommes ainsi pas attachés à produire une analyse des événements de gafieira, de samba rock et de ceux qui se produisent dans les bars sophistiqués; qui par eux-mêmes donneraient lieu à un autre article.

Cette «manière» adoptée pour traiter les réseaux de socialité présents dans le «monde de la samba», nous a permis de ne pas être attachés à la conception classique du territoire comme puissance territoriale.

À cet égard, la connaissance des territoires de la samba a ouvert la possibilité de comprendre les relations avec d'autres lieux en élargissant le débat au-delà de la sphère de la culture et en y insérant d'autres dimensions, liées à la vie quotidienne. C'est en effet à partir de leur vie quotidienne que les sambistas définissent leur territoire et font usage de ce qui précède la notion de territoire - l'espace géographique.

\footnotetext{
3 “constituindo parte de seu equipamento sócio psíquico, adquirido em seu processo de socialização e de vida".

4 “pode ser construída a partir de múltiplos veículos, imaginários, sentimentos, posses, propriedades, usos, sem que nenhum deles signifique sempre o exercício efetivo de um controle sobre os objetos e as práticas sociais que aí ocorrem".
} 
Ce sont ces appropriations de l'espace géographique qui transforment celui-ci en territoires de la samba, appropriations assumées comme médiation de représentations construites à partir d'un imaginaire lié à la samba, où les propres quartiers favorisent des représentations de la vie urbaine. Non seulement les quartiers mais également les casernes et hangars des écoles de $s a m b a^{5}$, les viaducs adaptés aux répétitions, les arrière-cours, les terrasses de simples maisons, les centres culturels, les places publiques, les bars, les foires et les rues où se trouvent les cercles de samba. Plus que de simples bâtiments ou zones situées quelque part dans la ville, ces endroits acquièrent une diversité de significations et de valeurs subjectivement conçues et territorialisées.

On peut noter que la samba agit comme une des pratiques matérielles et symboliques qui contribuent au bien-être à São Paulo, et ce, même si la logique de concentration territoriale de la production culturelle est souvent liée à des facteurs économiques. Il y a un «monde de la samba » à São Paulo qui est basé sur la relation entre deux logiques complémentaires: la ville qui transforme une partie de la samba en un produit destiné à la consommation (notamment via le carnaval télévisé) et la ville des territoires musicaux de la samba, traditionnels points de l'entretien et de la configuration des liens d'appartenance et de sociabilités communautaires.

De cette façon, le carnaval, événement public et privé, tenu, pratiqué et estimé par les sambistas, ne se révèle pas comme étant le seul moment privilégié pour approfondir les réflexions. En effet, les autres événements qui se déroulent dans la ville tout au long de l'année sont d'une toute aussi grande importance, bien qu'ils soient peu considérés par les chercheurs qui étudient le sujet. Ces chercheurs, en considérant la samba dans son unique association avec le temps du carnaval, la réduisent à la logique de reproduction des rationalités programmées et mercantilisées ayant cours au sein du carnaval médiatique.

Par conséquent, la samba a commencé à s'exposer dans les sambodromes, cooptée par une industrie qui a troqué les fourrures animales des tambours par des fourrures synthétiques produites en quantités considérables.

\footnotetext{
${ }^{5}$ Les casernes des écoles de samba sont des espaces fermés où les répétitions ont lieu avant la parade du carnaval. Les hangars sont les lieux que les écoles de samba et les blocs du carnaval utilisent pour la fabrication de leurs costumes et de leurs «allégories», ainsi que pour la construction des chars carnavalesques. Dans ces lieux, les organisateurs du carnaval guident les couturières, les constructeurs de chars et les décorateurs tout au long des étapes de travail nécessaires à la parade du carnaval.
} 
Au sein des territoires musicaux de la samba, se produit un dialogue entre la tradition et la modernité, tradition qui est dynamique et réinventée chaque jour, mais pas nécessairement ruinée par la transformation des manifestations culturelles en spectacle, comme s'il y avait une perte inexorable de la tradition face à des processus de transformation culturelle.

Il faut rappeler qu'il y a des relations d'échanges qui ne sont pas commerciales, bien que tout commerce soit un échange qui utilise de la marchandise. Il est à noter aussi que, malgré le processus de mondialisation, tout ne se mondialise pas, dans la mesure où les localismes et les régionalismes sont renforcés par des processus culturels.

La samba s'est consolidée dans la ville de São Paulo à partir de la matrice rurale de la campagne, qui, associée aux premiers ranchos et cordons carnavalesques ${ }^{6}$ dans le contexte urbain pauliste, a rendu possible l'émergence des écoles de samba.

Nous croyons que les sujets de recherches samba et carnaval devraient toujours être traités en évitant le recours à un discours «romantique». Toutefois, il est indéniable que, d'un point de vue ritualiste, la samba permet de créer des sensations ou «états d'esprit» imprégnés d'expériences personnelles et profondes et capables de susciter des sentiments de joie et de renouvellement, bien que pour le sens commun la samba s'est vue réduite à un simple style musical et à l'un des principaux symboles de la représentation brésilienne à l'intérieur et hors du pays.

Nous pouvons considérer la samba comme une marchandise, un produit d'exportation brésilien, notamment depuis le phénomène Carmen Miranda. Cependant, la samba transcende ce sens. On peut la comprendre comme une façon de penser, de sentir et de construire des territorialisations privées dans la ville. Par conséquent, la samba est également synonyme de source d'inspiration, de rythmicité, d'enjouement, de puissance créatrice et libertaire, d'« exercice religieux » pour certains et d'activité poétique et révolutionnaire pour d'autres.

Nous pouvons associer à ces réflexions le fait que les territoires de la samba sont des territoires concrets et/ou symboliquement appropriés à une signification qui va audelà de leurs limites physiques et de leurs possibles utilisations matérielles.

\footnotetext{
${ }^{6}$ Désignations utilisées entre les années 1910 et 1960 pour désigner les groupes de fêtards qui, pendant le carnaval, dansaient et chantaient dans les rues au son des instruments musicaux.
} 
En général, quand on parle de «samba de racine », nous utilisons des références de nature historique. Ainsi, la prédominance est donnée à l'histoire générale de la samba et aux productions des grands maîtres-compositeurs. Toutefois, pour les sambistas qui fréquentent les mouvements de samba ou les écoles de samba, existe une base historique mieux organisée et basée sur la connaissance transmise par leurs propres parents ou amis. D'une certaine manière, les mouvements de samba sont une réaction à la samba vendue comme produit commercial et soumise aux «engrenages» de l'industrie culturelle.

Une grande partie de ce qui est transmis par la «culture de la samba » est située dans le domaine de l'oralité et non dans le domaine de l'alphabétisation (qui tend à créer une forme de discipline et à organiser les territoires autours de logiques fonctionnelles et bureaucratiques). Dans certains cas, l'oralité peut fonctionner comme source de déviation, en créant une résistance aux puissances qui constituent et forment les territoires du pouvoir, de la discipline, de l'administration et de la bureaucratie. Toutefois, la «culture de la samba» ne se situe pas à l'extérieur de la dynamique apportée par la modernité, mais s'inscrit dans une appropriation quotidienne du moderne, comme on peut le voir dans les compositions d'Adoniran Barbosa.

Dans la mesure où nous avons commencé à participer à des événements liés à la samba, nous nous sommes sensibilisés à la question de l'assistance mutuelle et à celle de l'amitié (bien que, celles-ci coexistent avec des conflits et des contradictions inhérents aux relations sociales). Il convient de noter que cette sociabilité typique du «monde de la samba » est un facteur particulier de vie et de joie au sein des classes populaires.

En outre, en essayant de comprendre les relations d'appartenance communautaire se jouant dans la ville de São Paulo, en se basant sur le sentiment d'adhérence à une localité, nous nous sommes rendus compte que l'approche communautaire est incapable de traduire, de par l'influence des hypothèses théoriques précédentes, la circulation, la coexistence et le «déracinement communautaire» présents dans les contextes métropolitains actuels. 


\section{Les Territoires Musicaux de la Samba en Europe}

Les représentations du Brésil ont été produites et reproduites depuis des siècles, dès la lettre de Pero Vaz de Caminha, contribuant ainsi à la création d'une image idéalisée positive ou stéréotypée négative du peuple brésilien. Ces représentations sont également présentes dans la musique brésilienne, celles-ci ayant été encouragées à partir du moment où l'État est devenu le promoteur majeur d'une image spécifique du Brésil, ce qui a conduit à l'utilisation médiatique d'une icône importante de l'identité brésilienne: la samba.

On pourrait se rappeler qu'historiquement, dans le gouvernement de Getulio Vargas (État Neuf), la samba a été reconnue comme la principale manifestation de la culture populaire brésilienne, représentant légitimement l'identité nationale. Dès ce moment, la samba a été nationalisée et a reçu le statut de «manifestation culturelle légitime », sous la vigilance des pouvoirs constitués:

À ce moment, a été mise en place la légende à propos de ce qu'était la culture populaire ou folklore et, à partir de là, elle a commencé à tout contrôler (...) Cela peut également être appliqué au cas de l'organisation des défilés carnavalesques (...). Dans quelle mesure les initiatives telles que la création de Sambódromos ne permettent-elles pas d'affecter la spontanéité et la créativité de la samba? (Francisco Rocha, témoignage recueilli par Alessandro Dozena le 19/10/2007 dans la ville de São Paulo - Brésil).

Actuellement la promotion du Brésil à l'étranger est principalement réalisée par le Ministère du Tourisme, avec l'utilisation de clichés connus liés à la samba, au carnaval et au football, comme le note Ortiz (2013):

La culture apparaît comme un outil pour stimuler le commerce. L'État devient ainsi un élément actif dans la promotion de "brasilitude". Il encourage, prévoit des moyens privés, récolte les intérêts issus de ces ressources et approuve les produits affichant les icônes de l'identité brésilienne. Tel est l'objectif de la "marque Brésil" dans le cadre du Ministère du Tourisme dont l'intention est de promouvoir le pays en direction des marchés étrangers (Ortiz, 2013, p. 630, notre traduction $)^{7}$.

\footnotetext{
7 “A cultura surge como instrumento para impulsionar o comércio. O Estado torna-se assim um elemento ativo na promoção da brasilidade, ele incentiva, provê aos interesses privados meios e recursos, e chancela os produtos com os ícones da identidade brasileira. Este é o objetivo da "marca Brasil" vinculada ao Ministério do Turismo, sua intenção é promover o país no mercado exterior” (Ortiz, 2013, p. 630).
} 
On sait qu'au Brésil, les différentes régions produisent une multiplicité de manifestations culturelles. Cette diversité est une caractéristique identitaire importante qui, bien qu'elle soit parfois négligée et inconnue dans notre propre pays, attire l'intérêt et nourrit l'imaginaire utopique à l'étranger.

C'est ce qui se produit à l'occasion du Samba-Festival dans la ville de Cobourg, où se rassemblent les Batucadas venant de différents pays européens ainsi que différents groupes de capoeira, qui allient musiques et danses brésiliennes. On peut noter que la dimension de l'identité peut servir d'instrument de légitimation ou d'affirmation des territoires politiques et en même temps s'intègre à un imaginaire géographique transnational qui perçoit la culture brésilienne comme étant entrainante. Ces pratiques contribuent à la diffusion des imaginaires et symboles territoriaux, actionnant et projetant une réalité qui est renforcée par les images et imaginaires, dans les représentations pré-conçues ${ }^{8}$ (notre traduction).

Le Samba-Festival est la plus grande fête de samba en Europe et se déroule chaque année en juillet, comme l'explique notre interviewée:

Cette fête rassemble une bonne partie des sambistas en Europe. Quand elle a été créé, elle regroupait presque toutes les écoles de samba et Batucadas qui existaient en Europe, mais il y a actuellement un filtrage en raison de l'énorme quantité de groupes européens intéressés. L'événement rassemble les écoles de samba et les Batucadas inspirés par les écoles de samba de Rio de Janeiro et par les rythmes brésiliens. C'est quelque chose de formidable, l'organisation allemande peut rassembler environ deux mille personnes et la samba s'approprie la ville, la transformant en véritable Cité de la Samba. C'est la plus grande fête de la samba dans le monde, en dehors du Brésil bien-sûr (Dudu, directeur de l'École de Samba Aquarelle, témoignage recueilli par Alessandro Dozena le 06/10/2015 dans la ville de Paris, France).

Cet evènement confirme la tendance actuelle qui est à l'organisation de festivals européens dans des contextes urbains, festivals capables de générer la construction de l'identité des villes où ils se déroulent et de convertir ces événements publics et/ou privés en attractions touristiques. En reconnaissant les manifestations culturelles dans les différents pays et en valorisant le patrimoine matériel et immatériel, la politique mise en place par l'Unesco va dans cette direction.

\footnotetext{
8 Pour plus d'informations, visitez le site: http://www.samba-festival.de/ < Consultation en 28/08/2018>.
} 
Dans le Samba-Festival, la réunion de Batucadas venant de divers pays européens provoque certainement une influence réciproque à partir de la confluence de différentes matrices culturelles. Les Batucadas sont des groupes de percussionistes formés de 10 à 40 personnes en moyenne, qui occupent et parcourent les rues des villes lors des événements festifs européens. Ce phénomène musical « accompagne la diffusion de la pratique de la percussion brésilienne dans le monde entier » (Vaillant, 2009, p.179) et dans le cas français, a été semé par une brésilienne:

En ce qui concerne l'origine des Batucadas en France, une personne a été fondamentale : Nicia Ribas D'Ávila. Elle a défendu sa thèse de doctorat en 1987, sur la sémiotique de la musique. Elle avait fait une thèse au Brésil sur l'école de samba Padre Miguel, plus précisément sur la "paradinha" du maître André. Sa thèse était que tout le monde peut apprendre à jouer de la samba dans une Batucada. Elle a fait de nombreux ateliers de $s a m b a$, à l'époque, quand elle était ici en France, elle a créé sa propre école de $s a m b a$, à la fois pour gagner sa vie comme pour illustrer sa thèse, défendant le fait que tout gringo ${ }^{10}$ pourrait jouer de la samba, à partir de la subdivision du rythme de la samba dans les cellules de base et dans leurs différents instruments. Dans ces ateliers, elle a planté des graines dans toute la France, en Allemagne, en Finlande, en Angleterre, en Italie, en Espagne, parmi d'autres pays. Elle était une femme charismatique et contagieuse, elle est devenue une gourou de la samba en Europe, quelque chose d'impressionnant. Je l'ai rencontrée lors d'un atelier de tambours dans la Maison du Brésil, elle m'a donné une agogo à jouer, je ne pensais jamais jouer dans une Batucada. Après je suis entré dans son école de samba. (Dudu, directeur de l'École de Samba Aquarelle, témoignage recueilli par Alessandro Dozena au 06/10/2015 dans la ville de Paris, France).

L'étudiante mentionnée par l'interviewé, Nicia Ribas D'avila, reçoit le titre de docteure en Sciences du Langage à l'Université Sorbonne Nouvelle, pour sa thèse intitulée «Approche Sémiotique du Fait Musical Bresilien Batucada ». Selon les rapports des personnes interrogées, elle a été l'une des pionnières dans l'inclusion de la samba jouée par les Batucadas et ce, en utilisant les mêmes instruments de musique que les batteries des écoles de samba du Brésil, dans le but de motiver le développement des habiletés motrices chez les participants des Batucadas ${ }^{11}$.

\footnotetext{
${ }^{9}$ Il s'agit du moment au cours duquel il y a une pause dans le rythme joué, suivi par l'exécution rythmique par l'instrument musical appelé « repinique », avant le retour de l'école de samba ou Batucada.

${ }^{10}$ Terme utilisé au Brésil pour faire référence à des étrangers.

${ }^{11}$ Pour plus d'informations voir: http://www.niciadavila.com.br/
} 
En réfléchissant aux expériences concernant le tourisme musical, la polarisation du Brésil sur la scène internationale est irréfutable. On peut voir par exemple l'attraction que certaines villes brésiliennes exercent sur les percussionnistes européens:

Le tourisme musical à Recife - et dans sa ville historique adjacente Olinda - est récent, en comparaison à Salvador ou Rio, ce qui fait émerger depuis quelques années de nombreux débats au sein des communautés musicales récifiennes sur le statut de la musique de maracatu et sur sa diffusion dans le monde. Les batucadas arrivant à Recife ont pour la plupart déjà effectué un voyage à Rio ou à Salvador les années précédentes, et connaissent plus ou moins bien les structures musicales des samba et samba-reggae. L'initiation au maracatu, que ces groupes recherchent, implique l'apprentissage de codes rythmiques très différents qui sont joués sur des instruments encore peu répandus au sein du réseau international des batucadas" (Vaillant, p.185, 2009).

Les raisons qui me poussent encore aujourd'hui à approfondir mon savoir et mon savoir-faire dans la musique brésilienne sont la puissance et la créativité de ces musiques aux accents d'Afrique et l'intérêt pour la culture brésilienne métissée. Pour moi la force de ces musiques vient des rythmes des percussions qui sont très différents en fonction des régions (samba à Rio et São Paulo, samba-reggae à Salvador, frevo et maracatu à Recife, forro nordestinien etc.). Ces rythmes sont très puissants et ils possèdent un swing dansant qui vient d'une tradition en perpétuelle évolution. Cette musique populaire qui se transmet et évolue de génération en génération à une âme vivifiante et très vraie par rapport à des musiques occidentales que je trouve plus moroses. Les chansons, les sambas du sud-est brésilien ont aussi cette force de venir du passé tout en incorporant des nouvelles chansons. C'est une vraie culture populaire qui manque en Europe ou qui a disparu car les musiques européennes manquent de cette force et de ce swing qui, pour moi, vient d'Afrique" (Olivier Plouchard, témoignage recueilli par Alessandro Dozena, 25/04/2016 dans la ville de Tours - France).

La recherche de l'intégration de la culture brésilienne dans le corpus culturel européen présuppose la reconstruction de la culture d'origine puisque, dans cet échange, des concessions sont faites par la culture foránea, dans ce cas le Brésil. En ce sens, nous sommes d'accord avec Ortiz pour affirmer que «le pays Brésil peut être présenté comme local, régional, national et mondial ${ }^{12}$ notre traduction» et que son insertion dans le monde permet de telles variations, que «chacun [...] peut mettre en œuvre [...] ce qu'il souhaite promouvoir ${ }^{13}$ notre traduction» (Ortiz, 2013, p. 631).

\footnotetext{
12 “o país Brasil pode ser desta forma declinado como local, regional, nacional e global”.

13 “cada uma delas encontra-se operacionalizada em função do que se quer promover”.
} 
Et même sans vouloir promouvoir le tourisme ou l'image du pays, l'influence rythmique brésilienne sur les batucadas françaises sonne avec intensité:

La batucada fait maintenant partie du paysage culturel français, en apparaissant dans les carnavals de villes et villages, dans les fêtes et festivals partout en France. En même temps, son appropriation par les groupes (qu'ils soient spécialisés dans un style particulier ou qu'ils soient plus généralistes) fait que dans le répertoire, dans la forme ou même dans les instruments utilisés, on ne peut plus vraiment parler de samba dans certains cas, mais plutôt de rythmes d'inspiration brésilienne. Le phénomène inverse, marginal à mon sens, existe cependant aussi: les nombreux français et européens qui vont régulièrement au Brésil (je pense en particulier à ceux qui comme moi vont à Rio de Janeiro) sont en contact avec les musiciens et les chefs de batteries cariocas, au travers de stages et de cours, et à ce titre peuvent avoir une influence - certes minime - sur les mestres, en leur apportant des idées ou des pistes d'innovation (Alexandre Nouvel, témoignage recueilli par Alessandro Dozena le 25/10/2016 dans la ville de Toulouse, France).

Il est donc à noter une construction identitaire mise en évidence et développée à partir de la musique brésilienne. Cela démontre une énorme capacité à se référer à des modèles culturels partiellement mondialisés mais toujours étroitement liés aux contextes locaux et nationaux. Nous notons également que l'identité musicale brésilienne ne reste pas limitée au territoire brésilien, mais génère une appartenance mondiale fluide et déterritorialisée, acculturée à d'autres musiques et contextes culturels. Nous pouvons alors voir le développement d'identités locales qui introduisent ou mettent l'accent sur l'identité musicale brésilienne dans des lieux différents. Voilà ce que dit notre interlocutrice à propos de la présence de certains éléments culturels brésiliens en Espagne:

Je pense qu'en Espagne la culture brésilienne est connue pour sa grande variété de styles musicaux tels que la samba, la bossa-nova, le forró. Des chansons comme "Girl from Ipanema" sont chantées par tous les espagnols. Mais la culture du carnaval, de la samba et surtout la culture du football sont aussi bien connues. Je pense que cela ressemble à une fusion des cultures qui mêle le populaire traditionnel avec le contemporain. ça ressemble à une culture carnavalesque de rue (Silvia Mas, percussionniste de la Batucada Markatú Sitges - Espagne, témoignage recueilli par Alessandro Dozena le 02/04/2016 dans la ville de Montpellier - France) ${ }^{14}$.

\footnotetext{
${ }^{14}$ Creo que en España la cultura brasileña es conocida sobre todo por su gran variedad de estilos musicales como la samba, la bossa-nova, el forro. Canciones como "Garota de Ipanema" es tarareada por todos los españoles. Pero se conoce muy bien también la cultura del carnaval, la samba y sobretodo la cultura del futbol. Creo que se ve como una fusión de culturas que mezcla lo popular con lo más contemporáneo. Se ve como una cultura callejera y carnavalesca.
} 
Les motivations à la formation spontanée de Batucadas dans plusieurs pays européens, ainsi que la tentative de reproduction de rythmes semblables à ceux joués au Brésil s'expliquent de la façon suivante par Vaillant:

Il ne s'agit plus seulement de jouer : les participants travaillent collectivement à reproduire les musiques à l'identique, le plus fidèlement possible, jusqu'aux chants en portugais, aux costumes, aux danseuses, voire jusqu'à l'organisation sociale des écoles de samba. Cette reproduction n'exclut pas la composition, les chants en français, voire des innovations tout en conservant les codes musicaux « traditionnels », mais la création musicale reste un épiphénomène. Les groupes se spécialisent alors dans une famille musicale (samba, sambareggae, maracatu) puis choisissent un ou plusieurs groupes brésiliens comme modèles de la pratique musicale. Cette forme d'appropriation orthodoxe, ou brésilianiste, est minoritaire en France, mais plus répandue dans d'autres pays comme par exemple l'Angleterre, la Finlande ou le Japon (Vaillant, 2009, p. 190).

La présence d'éléments de la culture brésilienne avance avec force en Europe, influençant les fêtes carnavalesques européennes et renforçant la force attractive du pays face aux yeux étrangers. Cela peut être vu dans la Carte 1 qui montre l'intérêt significatif des français pour les cours de samba (cours de danse et de musique liés à la $s a m b a)$ et la distribution régionale de ces cours, présents dans pratiquement toutes les régions du pays: 


\section{Carte 1 - Distribution Régionale des Cours de Samba en France}

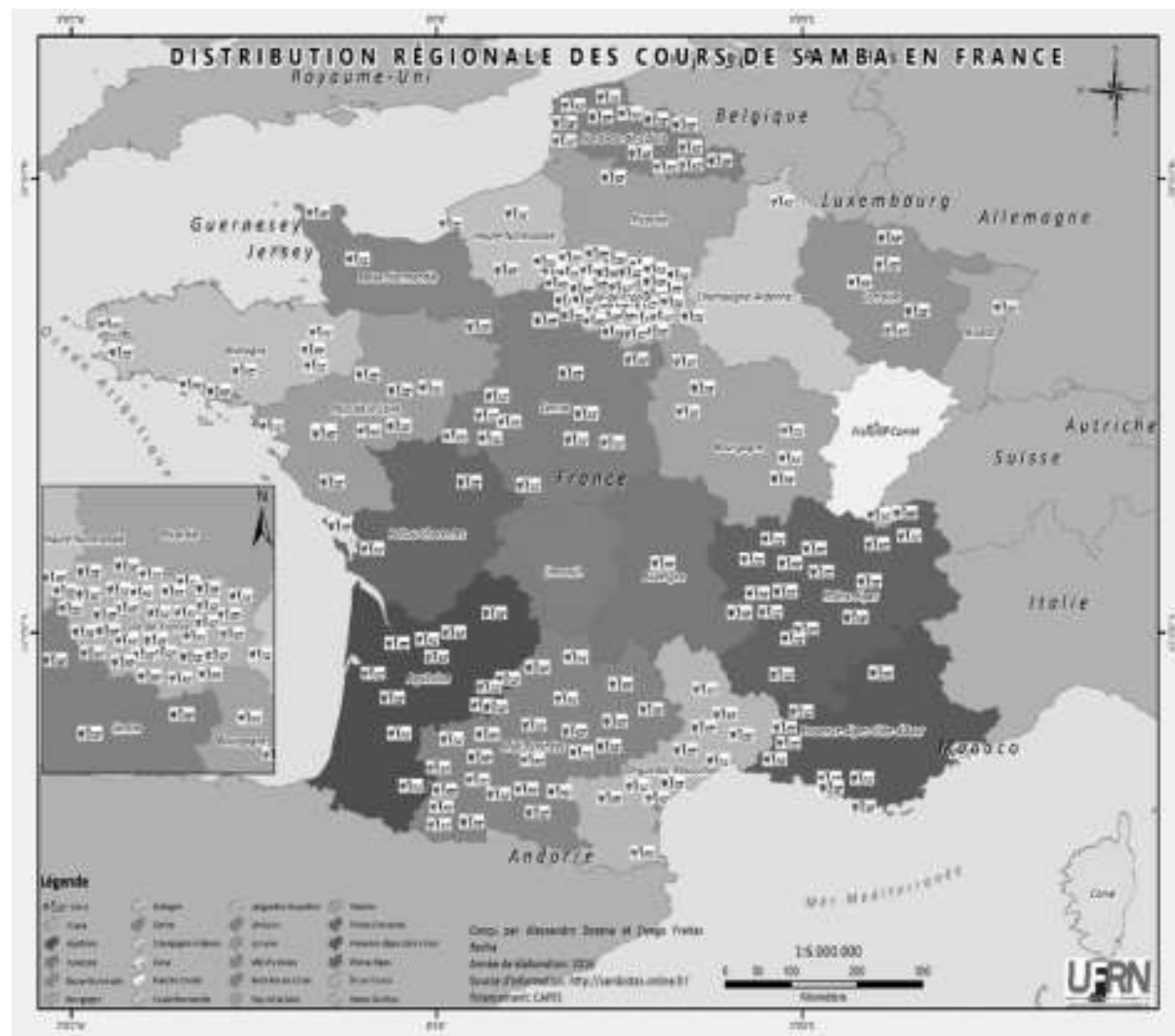

Source: Conçue par Alessandro Dozena, 2016.

De même, l'intérêt pour les cours de samba se retrouve dans d'autres pays européens $^{15}$, ce qui renforce également l'attrait touristique qu'exerce le Brésil.

\section{Considérations finales}

De la même façon que le flamenco étudié par Canova (2015), la samba a transmis sa forme de style musical et est aujourd'hui "reconnue comme un phénomène musical hors de toute norme, ayant une forte composante locale et connaissant une diffusion à travers le monde [...] par son lien avec les préoccupations contemporaines et son inscription dans le passé et le présent ${ }^{16}{ }^{»}$ (Canova, 2015, p. 471).

\footnotetext{
${ }^{15}$ Un cas illustratif est l'école de samba Masamba de Dublin - Irlande, qui présente et organise des ateliers dans les écoles, les universités, les clubs et les entreprises. Pour plus d'informations consulter: http://www.masamba.com/contact-us/ <access mai 2016>.

16 "reconocido como fenómeno musical fuera de toda norma por tener um fuerte componente local y conocer una extensa difusión por todo el planeta [...] por su vinculación con las preocupaciones contemporáneas y su inscripción en el pasado y el presente".
} 
Tout chercheur/chercheuse prêt(e) à explorer les territorialités de la samba, avec le souci de découvrir ses particularités phénoméniques à partir de la vie quotidienne des sambistas, entreverra d'énormes richesses, tant en ce qui concerne les moyens de structuration et d'utilisation du territoire, qu'au niveau des modes de vie des sambistas.

Dans le cadre de toutes les réflexions sur le «monde de la samba », nous avions comme « arrière-plan » les images et les scénarios perçus à travers nos expériences de terrain. Dans le cas de São Paulo, nous avons cherché à comprendre la ville comme une totalité dynamique et dialectique où les sambistas éprouvent dans leur vie quotidienne, avec intensité, les réseaux sociaux particuliers dans lesquels ils s'insèrent. En outre, ils développent dans leur imaginaire un point de référence identitaire avec leur ville mais aussi avec leur quartier.

La recherche sur le terrain a affecté sensiblement ma subjectivité en tant que résident d'une ville que je suis arrivé à mieux connaître. Il faut dire que la production d'un vidéo-documentaire a contribué à une meilleure compréhension de ma part des territorialités de la samba dans la ville de São Paulo, et m'a prouvé que les rationalités basées sur "l'imagination géographique" peuvent faire progresser la méthode scientifique et la théorie géographique. En fait, nous pensons que suivant les paramètres scientifiques et étant guidés par un découpage analytique clair, cet outil était adéquat pour capturer quelques connaissances pas toujours traduisibles seulement par des procédures plus artistiques de recherche de compréhension de la réalité ${ }^{17}$.

Après avoir approché les territoires musicaux de la samba en Europe, nous pensons que la samba peut être abordée comme un phénomène socio-spatial en mesure d'établir un lien entre la culture mondiale et l'identité brésilienne, à partir de dimensions imaginaires qui traversent l'expérience musical.

Ce débat nous permet d'agrandir le champ de réflexions et d'offrir la possibilité de mettre en évidence la dimension spatiale des phénomènes spatiaux liés à la musique et à des représentations imagées, stéréotypées ou non.

\footnotetext{
17 Ce documentaire peut être vu en deux parties, sur la chaîne YouTube: https://www.youtube.com/watch?v=S6ytZZxngOQ 
Notre objectif était de réfléchir aux représentations actuelles de la samba à l'étranger et de montrer qu'elles constituent des outils de valorisation, de promotion et de demande touristique, agissant sur l'imaginaire au travers de la musique brésilienne (principalement la samba) qui en est un vecteur puissant.

\section{Références}

Canova, N. (2015). La música como objeto geografico. Estado de la Cuestión y perspectivas de Tratamiento. In: Revista de Antropología Experimental n ${ }^{\circ}$ 15, Texto 26, p.465-482, 2015.

Certeau, M. de; Giard, L.; Mayol, P. (1997). A invenção do cotidiano: morar $e$ cozinhar. Petrópolis: Vozes, v. 2, 376p.

Crozat, D. (2016). Jeux et Ambigüités de la Construction Musicale des Identités Spatiales. In: Dozena, A. (dir.) Geografia e Música: Diálogos, Natal: Editora da UFRN. p. 13-57.

Dozena, A. (2012). A geografia do samba na cidade de São Paulo. São Paulo: PoliSaber, 264p.

Gomes, P. C. da C. (2002). A condição urbana: ensaios de geopolítica da cidade. Rio de Janeiro: Bertrand Brasil, 304p.

Moraes, A. C. R. (2001). Capitalismo, geografia e meio ambiente. Tese (Livre Docência) - Faculdade de Filosofia Letras e Ciências Humanas, Universidade de São Paulo, São Paulo. 202p.

Ortiz, R. (2013). Imagens do Brasil. In: Revista Sociedade e Estado. Setembro/Dezembro, Número 3, Volume 28, p.609-633.

Santos, M. (2002). A natureza do espaço: técnica e tempo, razão e emoção. São Paulo: Edusp, 384p.

Vaillant, A. (2009). Territoires de la batucada: circulations et appropriations d'une pratique musicale brésilienne. In: Raibaud, Yves (dir.). Comment la musique vient aux territoires. Pessac: Publications de la Maison des Sciences de L'homme d'Aquitaine, vol. 1, p.179-196. 